\title{
A follow-up audit of the completion of bone marrow specimen request forms at an academic laboratory
}

\author{
L Budding,' (D) MJ Coetzee, ${ }^{2}$ (D) G Joubert ${ }^{3}$ \\ 'Department of Anatomical Pathology, School of Pathology, Faculty of Health Sciences, University of the Free State \\ and National Health Laboratory Service, South Africa \\ ${ }^{2}$ Department of Haematology and Cell Biology, School of Pathology, Faculty of Health Sciences, University of the Free State \\ and National Health Laboratory Service, South Africa \\ ${ }^{3}$ Department of Biostatistics, School of Biomedical Sciences, Faculty of Health Sciences, University of the Free State, South Africa
}

Corresponding author, email: coetzeemj@ufs.ac.za

\begin{abstract}
Background: In order to ensure that patients receive individualised treatment following bone marrow biopsy, it is necessary for clinicians to provide complete clinical information on bone marrow request forms (BMRFs). An audit of BMRFs six years previously showed poor completion, especially with regard to filling in full blood count results, transfusion history, medication history, information about the clinical examination and HIV status. This lead the laboratory to design a new bone marrow specimen request form. We did a follow-up audit to see if the new form had helped to improve the completion rates.
\end{abstract}

Methods: We compared 400 forms to the 357 that were audited in 2013. The following details were recorded: date and time of collection, patient demographics, requesting doctor's details, clinical information, current medication, transfusion history and HIV status, and details of the procedure completed by technologists, registrars and pathologists.

Results: The 2019 follow-up audit showed significant improvements in the completion of the transfusion history, as well as the clinical examination and HIV status. Registrars and pathologists signed off forms regularly. The completion of patient demographic details, and requesting doctors' names and telephone numbers worsened.

Discussion and conclusion: We recommend that the form be simplified so the requesting doctors only need to tick yes or no, in a tick-box format, if a full blood count has been done in the preceding 24 hours. There needs to be a dedicated space for the hospital and laboratory stickers. Only the name and telephone number of one doctor should be requested. This doctor should preferably be the most senior doctor involved with patient care. All referring laboratories and hospitals will be consulted before updating the form. Unfortunately, it seems that the only way to force the completion of request forms is to introduce an electronic order entry system that does not accept incomplete forms.

Keywords: bone marrow examination, laboratory request forms, preanalytical phase, haematology, clinical audit

\section{Introduction}

Incomplete laboratory request forms remain a common preanalytical error in haematology., ${ }^{1,2}$ Properly completed bone marrow request forms (BMRFs) can guide haematologists to focus on a clinically relevant diagnosis and can help them to request appropriate additional tests. Coetzee et al. ${ }^{3}$ reported an audit of the completion of BMRFs received over a period of four months in 2013 at the Universitas Academic Laboratories of the National Health Laboratory Service (NHLS) in Bloemfontein. Full blood count $(\mathrm{FBC})$ results were absent on $57.7 \%$, the transfusion history on $29.1 \%$, the medication on $23.8 \%$, and information about the clinical examination and HIV status on $9.2 \%$ of forms. Inaccurate and incomplete reporting on specimen request forms may lead to negative patient outcomes ${ }^{4}$ and can contribute to increased turn-around times. In an attempt to encourage the completion of patient identifiers and clinical information, the laboratory introduced a new BMRF in 2013 (see Figure 1). It was distributed to all laboratories that refer bone marrow specimens to Bloemfontein after the users had been informed. The aim of this current update was to evaluate whether the new BMRF resulted in improved completion of the form.

\section{Methods}

To assess whether the new BMRF improved the completion, a follow-up audit was done on BMRFs submitted to the Universitas NHLS Haematology Laboratory over a period of four months in 2018. A retrospective, cross-sectional study was conducted. A sample of 400 request forms was analysed and compared to the 357 BMRFs from the 2013 audit. The completion of the following aspects were recorded:

- Date and time of collection of specimen.

- Patient demographics.

- Requesting doctor's details.

- Clinical information i.e. current medication, transfusion history and HIV status.

- Other laboratory tests requested.

- Details of the procedure completed by attending technologists. 


\begin{tabular}{|l|l|l|}
\hline \multicolumn{1}{|c|}{ BONE MARROW REQUEST FORM } \\
\multicolumn{1}{|c|}{ Department of Haematology and Cell Biology, UFS and NHLS; Tel (051) 405 3887 } \\
\hline Date: & Time: & Female \\
\hline Patient details (sticker if possible) & Male \\
\hline Surname, Name & & Hospital \\
\hline Date of birth & Ward \\
\hline Hospital no. (UM, PM, etc.) & \\
\hline
\end{tabular}

ICSH guidelines for the standardization of bone marrow specimens and reports. International Journal of Laboratory Hematology 2008;30:349-364.

No bone marrow will be processed without an accompanying FBC having been done in the last 24 hours!

\begin{tabular}{|c|c|c|}
\hline \multirow{2}{*}{$\begin{array}{l}\text { Has } \mathrm{FBC} \text { has been done in the last } 24 \mathrm{~h} \\
\text { Requesting consultant: }\end{array}$} & \multicolumn{2}{|l|}{ Signature of doctor } \\
\hline & MP number & Contact No: \\
\hline Requesting doctor: & MP number & Contact No: \\
\hline \multicolumn{3}{|l|}{ Clinical background: } \\
\hline \multicolumn{3}{|l|}{ Indication for bone marrow } \\
\hline \multicolumn{3}{|l|}{ Previous bone marrows? } \\
\hline \multicolumn{3}{|c|}{ Medication (e.g. current chemotherapy cycle, date, herbal medication, supplements) } \\
\hline \multicolumn{3}{|l|}{ Recent bleeding? } \\
\hline \multicolumn{3}{|c|}{ Recent Blood Transfusions (and pre-transfusion $\mathrm{Hb}$ ) } \\
\hline Splenomegaly? (cm) & Hepatomegaly? $(\mathrm{cm})$ & \\
\hline \multicolumn{3}{|l|}{ Lymphadenopathy? where and size $(\mathrm{cm})$} \\
\hline HIV status: & Negative & Not disclosed \\
\hline
\end{tabular}

BONE MARROW SPECIMENS IN ORDER OF DRAW
\begin{tabular}{|l|l|l|l|l|}
\hline & Test & Volume & Please Sign if obtained \\
\hline 1 & Compulsory & Aspirate slides & $0.5 \mathrm{~mL}$ & \\
\hline $\mathbf{2}$ & Compulsory & Purple (EDTA) (flow and molecular tests) & $1-2 \mathrm{~mL}$ & \\
\hline 3 & Compulsory & Green (heparin) (FISH) & $1-2 \mathrm{~mL}$ & \\
\hline 4 & Compulsory & Green (heparin) with medium for karyotyping & $1-2 \mathrm{~mL}$ & \\
\hline $\mathbf{5}$ & \multicolumn{1}{|c|}{ Optional } & TB/Fungal culture (in culture bottle) & $1-5 \mathrm{~mL}$ & \\
\hline $\mathbf{6}$ & Compulsory & Trephine & At least $2 \mathrm{~cm}$ (adults) & Length: \\
\hline $\mathbf{7}$ & Optional & Imprint if aspirate unsuccessful & & \\
\hline
\end{tabular}

Additional requests

Molecular and cytogenetic tests will be requested by pathologists once the specimen has been reviewed

\begin{tabular}{|l|l|l|}
\hline $\begin{array}{l}\text { FOR LAB USE ONLY } \\
\text { Samples received: }\end{array}$ & \multicolumn{2}{l|}{ Referral Laboratories: attach most recent FBC printout and PB smear } \\
\hline BM Asp Yes/No & Purple EDTA BM Yes/No & Cytogenetics FISH BM Yes/No \\
\hline Cytogenet. medium BM Yes/No & TB/Fungal culture Yes/No & Trephine Yes/No \\
\hline Trephine length (cm) & Imprint Yes/No & \\
\hline Notes: & Reported by: & Signed off by pathologist: \\
\hline Smears made by: &
\end{tabular}

Figure 1: New bone marrow request form

Results were summarised by frequencies and percentages and the two audits compared using chi-squared tests or Fisher's exact tests in the case of sparse cells. The categories were only considered to be complete if all the criteria were completed on the BMRF. Approval was obtained from the Health Sciences Research Ethics Committee of the University of the Free State (UFS-HSD2019/0325/2603) and laboratory authorities.
Results

The results are shown in Figure 2. The 2019 follow-up study showed the following improvements:

- Transfusion history was now only absent on $22.3 \%(P<0.0001)$.

- Clinical examination and HIV status were now absent on $1.3 \%$ of forms $(P=0.0029)$. 


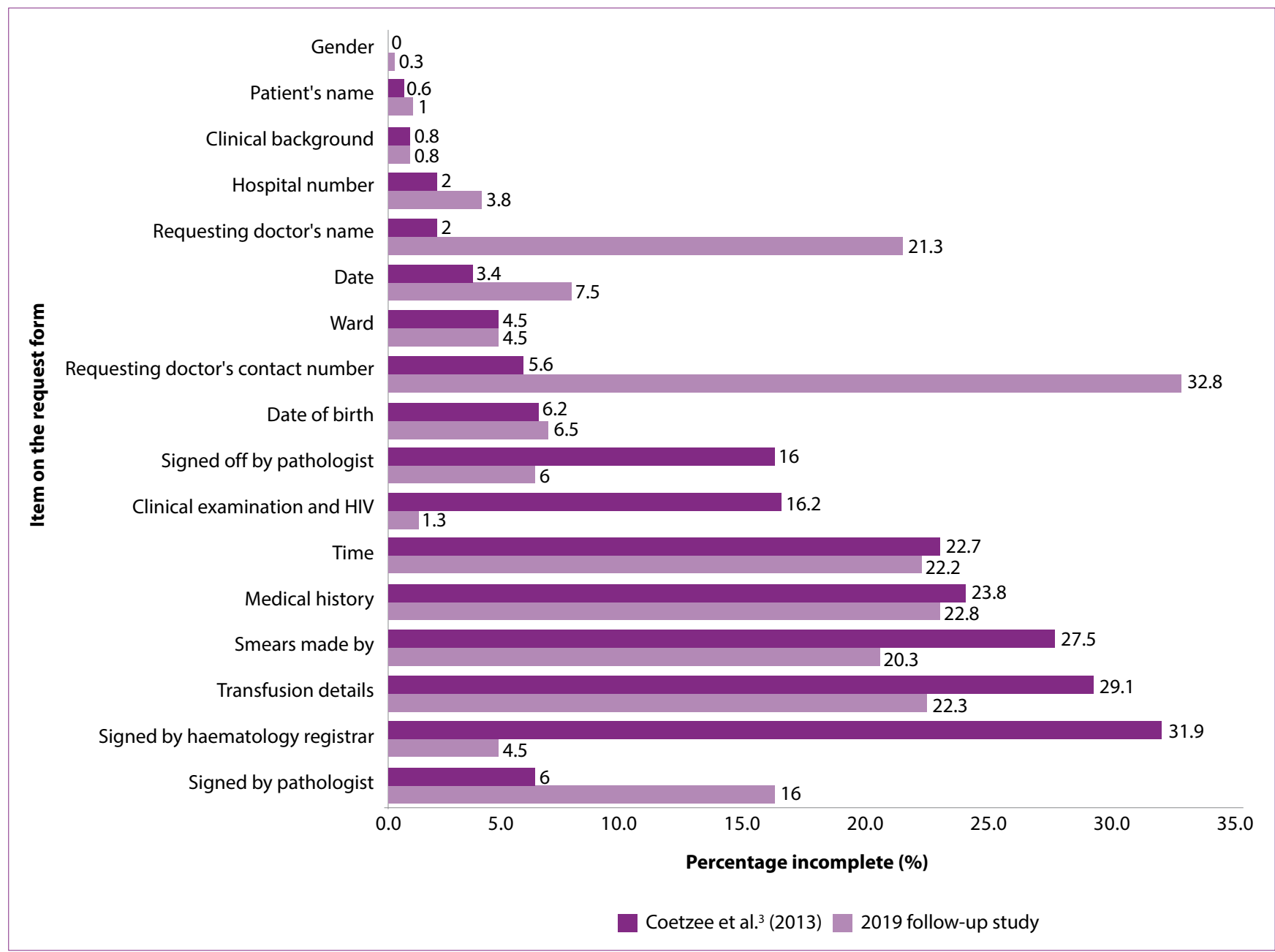

Figure 2: Comparison of percentage incomplete items on the request forms between the 2013 study (Coetzee et al. $)^{3}(n=357)$ and the follow-up audit in $2019(n=400)$. The order of the items on the $y$-axis follows their sequence on the request form.

- The proportion of forms lacking registrars' signatures decreased from $31.9 \%$ to $4.5 \%(P<0.0001)$.

- The forms without pathologist signatures decreased from $16.0 \%$ to $6.0 \%(P<0.0001)$.

Some laboratories modified the form so that requesting doctors only had to tick that a FBC was done in the last 24 hours. The pathologists could then retrieve the results from the laboratory information system. We, therefore, were not able to audit the completion of $\mathrm{FBC}$ results. The requests for additional tests were also too different to compare between the previous BMRF and the newly implemented form.

The space for patient stickers on the new form was not large enough, so that stickers often covered parts of the space for patient demographics. Sometimes doctors only used abbreviated stickers that did not include the patient's first name(s), date of birth or sex. These abbreviated stickers did, however, include the hospital number, which meant that the correct patient demographics were transferred from the hospital to the laboratory information system. The stickers often obstructed the space for the date of the specimen collection. It is necessary to know the time that the specimen was collected in order to determine whether the specimen is viable for flow cytometry or genetic investigations.
The doctor's name and contact details on BMRFs are required in case the laboratory has questions about the patient. Coetzee et al. $^{3}$ found that the BMRFs were often completed by junior doctors, who did not know enough about the patient to be able to answer questions from pathologists. They therefore added a space for the consultant's details on the BMRF. This part of the BMRF was poorly completed, with $32.8 \%$ of the forms having no contact details of either requesting consultant or requesting doctor, compared to $5.6 \%$ in $2013(P<0.0001)$. Similarly, the requesting doctor's name was absent from $21.3 \%$ of BMRFs, compared to $2.0 \%$ in $2013(P<0.0001)$.

\section{Discussion and conclusion}

To our knowledge these are the only reported audits of BMRFs. Due to the design of the new BMRF, some sections of the form were inadvertently poorly completed by clinicians. We therefore recommend that the following changes be made to the form:

1. The question regarding whether an FBC has been done in the last 24 hours should be presented in tick-box format with options for clinicians to tick "yes" or "no." The FBCs can then be retrieved from the laboratory system.

2. There should be dedicated spaces for both the hospital sticker and the laboratory barcoded stickers. 
3. Only one doctor's name and contact details (preferably the most senior doctor who would be able to answer pathologists' questions regarding the patient) should be requested.

We did not include an audit of the additional tests that were requested because the layout of that part of the new BMRF has changed significantly. At Universitas NHLS Haematology Laboratory, only haematologists and pathology registrars are allowed to request flow cytometry and cytogenetics when appropriate. This has reduced the number of unnecessary requests by clinicians.

Before the implementation of the next version of a request form, we will consult with more laboratories (in private and public), pathologists, clinicians and registrars than we had done previously. This will ensure better buy-in from all parties and will ensure an updated BMRF that is suitable for most haematology departments. The guidelines of the International Council for Standardization in Hematology (ICSH) ${ }^{5}$ and the South African National Pathology Group (NPG) 6 provide guidance concerning request forms. However, the only way to ensure that laboratory request forms are adequately completed is to introduce an electronic order entry system ${ }^{7}$ that will not accept incomplete request forms. Standard operating procedures for patient identification and specimen collection ${ }^{8}$ need to be promoted so that specimens comply with ISO 15189:2012 ${ }^{9}$ requirements.

\section{Acknowledgements}

We would like to thank the staff of the Haematology Laboratory and Laboratory Support Services of the Universitas Academic Business Unit, NHLS, for their support throughout this study. In particular, the staff of the Division of Clinical Haematology, and the Department of Haematology and Cell Biology, need to be thanked.

\section{Conflict of interest}

The authors have no conflicts of interest.

\section{Funding source}

There was no special funding for this study.

\section{Ethical approval}

Approval was obtained from the Health Sciences Research Ethics Committee of the University of the Free State (UFSHSD2019/0325/2603) and laboratory authorities.

\section{ORCID}

L Budding (iD) https://orcid.org/0000-0002-4284-1866

MJ Coetzee (iD https://orcid.org/0000-0003-2762-0966

G Joubert (iD https://orcid.org/0000-0002-3728-6925

\section{References}

1. Wijegunaratne DTE, Pradeepa NAH, Dolawatte AP, Razana MYF, Ranasinghe NN. An audit on blood picture request forms submitted to haematology laboratory in a tertiary care hospital in Sri Lanka. British Journal of Haematology. 2020;189(S1):63. Available from: https://onlinelibrary.wiley.com/ toc/13652141/189/S1. (Accessed 09 September 2020).

2. De la Salle B. Pre- and postanalytical errors in haematology. International Journal of Laboratory Hematology. 2019;41(Suppl 1):170-6. https://doi.org/10.1111/ ijlh.13007.

3. Coetzee MJ, Joubert G, Swart M, et al. An audit of the completion of bone marrow specimen request forms at an academic laboratory. Medical Technology SA. 2017;31(1):1-6. Available from: https://journals.co.za/content/journal/10520/ EJC-a9cd8943f. (Accessed 09 September 2020).

4. Oyelekan AA, Ojo OT, Olawale OO, et al. Pattern of completion of Laboratory Request Forms in a tertiary health facility. Annals of Health Research. 2018;4(2):155-61. https://doi.org/10.30442/ahr.0402-7-18.

5. Lee SH, Erber WN, Porwit A, Tomonaga M, Peterson LC. ICSH guidelines for the standardization of bone marrow specimens and reports. Int J Lab Hematol. 2008:30:349-64. https://doi.org/10.1111/j.1751-553X.2008.01100.x

6. National Pathology Group. National Pathology Group Coding Guideline: National Pathology Group; 2013. Available from: https://www.pathology. co.za/images/2013-12_NPG_Coding_Guidelines_V5191213.pdf. (Accessed 09 September 2020).

7. Nasir N, Shaikh MU, Ali N, Hussain S. Compliance of hand written transfusion requisition form and improvement after online request - a clinical audit. Journal of the Pakistan Medical Association. 2020;70(4):694-8. https://doi.org/10.5455/ jpma.14958.

8. Simundic A-M, Bölenius $K$, Cadamuro J, et al. Joint EFLM-COLABIOCLI recommendation for venous blood sampling. Annales de Biologie Clinique (Paris). 2019;77(2):131-54. https://doi.org/10.1684/abc.2019.1419.

9. International Organization for Standardization. Medical laboratories Requirements for quality and competence ISO 15189:2012. 3rd ed. Geneva: International Organization for Standardization; 2012. Available from: https:// www.iso.org/standard/56115.html. (Accessed 30 July 2020). 